\title{
Neuroimaging Evaluation for First Attack of Unprovoked Nonfebrile Seizure in Pediatrics: When to Order?
}

\author{
Hussain Sadeq ${ }^{a}$ Jumanah Karim $^{a}$ Yousef Marwan ${ }^{b}$ Talal AlSaleem ${ }^{c}$ \\ ${ }^{a}$ Department of Pediatrics, Al-Adan Hospital, ${ }^{\text {b}}$ Department of Orthopedic Surgery, Al-Razi Orthopedic Hospital, and \\ 'Department of Pediatrics, Mubarak Al-Kabeer Hospital, Kuwait City, Kuwait
}

\section{Key Words}

Neuroimaging studies $\cdot$ Pediatrics $\cdot$ Nonfebrile seizure

\begin{abstract}
Objective: To assess the value of neuroimaging studies in evaluating pediatric patients presenting with a first attack of nonfebrile seizure. Method: We reviewed the medical records of pediatric patients aged 28 days to 12 years who were admitted between 1 January and 31 December 2013 with a first attack of unprovoked, afebrile seizure. These patients had undergone neuroimaging studies. The exclusion criterion was patients with known predisposing conditions for seizure. The computed tomography (CT) scan and magnetic resonance imaging (MRI) results were either normal or abnormal, and the abnormal ones were further classified into clinically insignificant or significant. Descriptive analysis was performed to summarize the data. Result: Fifty children were identified with a mean age of $5.2 \pm 3.8$ years. Of the 50 subjects, 29 (58.0\%) were males and 21 (42.0\%) were females. Sixteen patients (32.0\%) had abnormal neuroimaging studies (CT scan, MRI or both); however, only 1 was considered to have a clinically significant abnormality, later diagnosed as Moyamoya disease. Conclusion: In this study, the neuroimaging studies were found not to be useful in evaluating pediatric patients presenting with a first attack of unprovoked, nonfebrile seizures.

(c) 2015 S. Karger AG, Basel
\end{abstract}

\section{Introduction}

Witnessing a child in seizure is an unpleasant experience for parents. The challenge is for the pediatrician to detect structural brain abnormalities associated with the seizure. Seizure among children is quite a common condition, accounting for $4-10 \%$ of all pediatric neurological disorders [1]. In the USA, it is estimated that $10 \%$ of the population will experience at least one attack of seizure once in their lifetime [2]. The importance of neuroimaging studies for pediatric patients presenting with nonfebrile seizure is still a debatable issue in the literature [3]. One meta-analysis study showed that findings on neuroimaging were abnormal in up to one third of children with a first attack of seizure; however, most of these abnormalities did not require any immediate medical or surgical intervention [4]. Focal neurological deficits and the presence of predisposing conditions to seizure among this group of patients are reported to be the strongest predictors of abnormal computed tomography (CT) scan findings $[5,6]$.

The current guidelines of the American Academy of Neurology (AAN) do not support the routine use of neuroimaging studies in such circumstances because of the lack of sufficient evidence of any benefit from such studies [4]. In Kuwait, there are no well-established guidelines regarding the use of neuroimaging studies for pediatric

\begin{tabular}{ll}
\hline KARGER & $\begin{array}{l}\text { ( ) 2015 S. Karger AG, Basel } \\
1011-7571 / 15 / 0251-0056 \$ 39.50 / 0 \quad \text { Karger }\end{array}$ \\
$\begin{array}{l}\text { E-Mail karger@karger.com } \\
\text { www.karger.com/mpp }\end{array}$ & $\begin{array}{l}\text { This is an Open Access article licensed under the terms of the } \\
\text { Creative Commons Attribution-NonCommercial 3.0 Un- } \\
\text { ported license (CC BY-NC) (www.karger.com/OA-license), } \\
\text { applicable to the online version of the article only. Distribu- } \\
\text { tion permitted for non-commercial purposes only. }\end{array}$
\end{tabular}

Hussain Sadeq

Department of Pediatrics

Al-Adan Hospital

Kuwait City (Kuwait)

E-Mail drhsadeq@icloud.com 


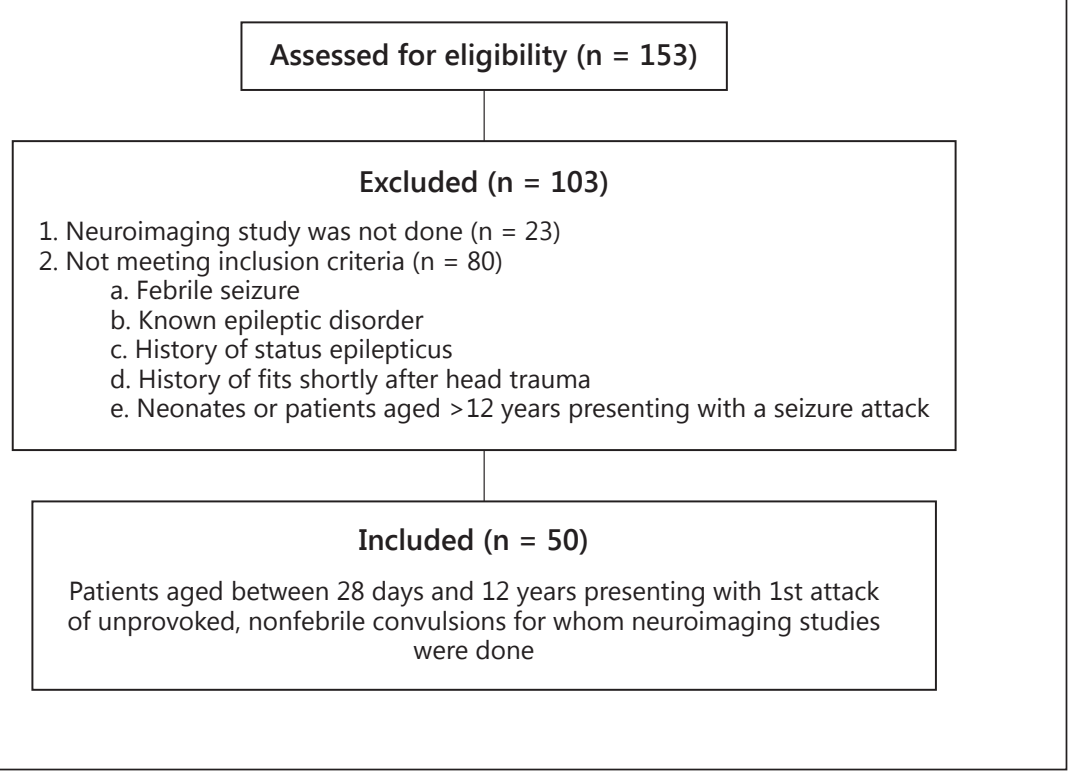

Fig. 1. Selection of patients.

patients with a first attack of nonfebrile seizure, although magnetic resonance imaging (MRI) has been found to be of value [7-9]. Emergency neuroimaging studies should be considered in any child of any age presenting with Todd's paresis $[10,11]$, and several investigations worldwide have addressed the importance of neuroimaging tests in evaluating pediatric patients with this presentation [12-14].

The aim of this study was to assess the usefulness of neuroimaging studies in pediatric patients presenting with a first attack of unprovoked, nonfebrile seizure.

\section{Materials and Methods}

This retrospective study was conducted at the Mubarak Al-Kabeer Hospital, Kuwait. The hospital was selected randomly from 5 general hospitals that have Departments of Pediatrics. The study involved pediatric patients aged between 28 days and 12 years, who were admitted for a first attack of unprovoked, nonfebrile seizure. It included patients admitted between 1 January and 31 December 2013.

The medical records of the patients were reviewed to identify those who had undergone neuroimaging. These radiological tests were done immediately following admission to the hospital; no patient was discharged before undergoing the studies. A consultant radiologist had assessed the images which were then evaluated by pediatric neurologists or neurosurgeons. During our chart review, provisional diagnosis of a first attack of unprovoked, nonfebrile seizure (as in the medical records) was considered if keywords such as epilepsy, fit, seizure, convulsion, afebrile, nonfebrile, ab- normal movement, loss of consciousness and fainting were written in the admission slip from the pediatric emergency room. Keywords such as abnormal movement, fainting and loss of consciousness were also considered as a provisional diagnosis because in some of the patients, such symptoms could be related to seizure disorders. Exclusion criteria were subjects with febrile seizure, a known epileptic disorder, a history of status epilepticus or fits occurring shortly after head trauma as well as neonates or patients older than 12 years. Any patient who did not have a neuroimaging test as part of the evaluation of the seizure was excluded (fig. 1).

The medical records were thoroughly examined to retrieve data regarding the demographic characteristics and features of the seizure of each patient. This included gender, age, the type and duration of the seizure, the presence of chronic disorders, the family medical history, exposure to toxins and developmental history. In addition, physical examination findings, investigations and imaging results were documented. Seizure types (i.e. partial or generalized) were defined based on the latest guidelines of the International League against Epilepsy [15]. The CT scan and MRI results were considered either normal or abnormal by the consultant radiologist, pediatric neurologist and pediatric neurosurgeon. Abnormal neuroimaging studies were further classified as clinically insignificant or clinically significant based on the need for immediate medical or surgical intervention.

This study was reviewed and ethically approved by the Review Board of the Ministry of Health of Kuwait. Moreover, permission to review and collect data from the patients' records was obtained from the administration of the hospital involved in our study. The collected data were entered and analyzed using the Statistical Package for Social Sciences (SPSS v17.0, IBM Inc., Chicago, Ill., USA). Frequencies and proportions were used to describe the demographic, clinical and neuroimaging data of the patients, which were then summarized in the form of tables. 
Table 1. Demographic characteristics and history of the participants

\begin{tabular}{|c|c|}
\hline Characteristics & $\mathrm{n}(\%)$ \\
\hline \multicolumn{2}{|l|}{ Gender } \\
\hline Male & $29(58.0)$ \\
\hline Female & $21(42.0)$ \\
\hline \multicolumn{2}{|l|}{ Age } \\
\hline $1-6$ months & $7(14.0)$ \\
\hline $6-12$ months & $3(6.0)$ \\
\hline $1-5$ years & $18(36.0)$ \\
\hline $5-12$ years & $22(44.0)$ \\
\hline Mean \pm SD & $5.2 \pm 3.8$ \\
\hline \multicolumn{2}{|c|}{ Developmental history } \\
\hline Normal & $45(90.0)$ \\
\hline Motor delay & $2(4.0)$ \\
\hline Global delay & $3(6.0)$ \\
\hline \multicolumn{2}{|l|}{ Family history } \\
\hline Yes & $20(40.0)$ \\
\hline No & $30(60.0)$ \\
\hline \multicolumn{2}{|l|}{ Seizure type } \\
\hline Focal & $17(34.0)$ \\
\hline Generalized & $33(66.0)$ \\
\hline \multicolumn{2}{|l|}{ Seizure duration } \\
\hline$<5 \mathrm{~min}$ & $32(64.0)$ \\
\hline $5-30 \min$ & $18(36.0)$ \\
\hline \multicolumn{2}{|l|}{ Chronic conditions } \\
\hline Yes & $11(22.0)$ \\
\hline No & $39(78.0)$ \\
\hline \multicolumn{2}{|l|}{ Toxin exposure } \\
\hline Yes & 0 \\
\hline No & $50(100.0)$ \\
\hline
\end{tabular}

\section{Results}

During the study period, 153 children were admitted with a first attack of nonfebrile seizure. Of these, 50 (32.7\%) met the inclusion criteria, i.e. 29 males (58.0\%) and 21 females (42.0\%; table 1). Their mean age was 5.2 \pm 3.8 years, with 40 children $(80 \%)$ between 1 and 12 years old and 10 children $<1$ year $(20 \%)$. Global or motor delay was noticed in 5 patients $(10.0 \%)$. Of the 50 children, $11(22.0 \%)$ had a history of preexisting chronic conditions; 6 of these were preterm patients, and the remaining 5 were cases of neonatal hypocalcemia, attention-deficit hyperactivity disorder, glucose-6-phosphate dehydrogenase deficiency, autism spectrum disorder and recurrent otitis media. Moreover, a first- or seconddegree positive family history of epileptic disorder was noted in 20 patients (40.0\%). Thirty-three patients (66.0\%) presented to the Emergency Department with generalized tonic-clonic seizures and 17 (34.0\%) present-
Table 2. Findings of the physical examination

\begin{tabular}{lc}
\hline Physical examination findings & $\mathrm{n}(\%)$ \\
\hline Mental status & \\
$\quad$ Normal & $49(98.0)$ \\
$\quad$ Disturbed & 0 \\
LOC/coma & \\
Dysmorphic features & $2(4.0)$ \\
$\quad$ Yes & $48(96.0)$ \\
No & $2(4.0)$ \\
Neurological skin features & $48(96.0)$ \\
$\quad$ Yes & $3(6.0)$ \\
No & $47(94.0)$ \\
Focal neurological deficit & \\
Yes & \\
No & \\
\hline LOC $=$ Loss of consciousness. & \\
\hline
\end{tabular}

ed with focal seizures. In addition, 32 (64.0\%) children presented with a brief $(<5 \mathrm{~min})$, unprovoked, nonfebrile seizure.

The findings of the physical examination are shown in table 2 ; 1 patient $(2 \%)$ was admitted to the hospital with a disturbed level of consciousness and a lethargic appearance, dysmorphic features were noted in $2(4 \%)$ and 2 others (4\%) had evidence of significant skin features (i.e. hypopigmented macules and café-au-lait spots; both of these patients had normal brain MRI results). Subtle or gross focal neurological deficits at the time of admission were noted in 3 patients (6\%) only.

The findings of the investigations and neuroimaging studies are summarized in table 3 . An elevation in white blood cell count was noted in 4 patients (8\%), while serum calcium abnormal levels were noted in $5(10 \%)$. Lactate and/or amino acid level tests were ordered for 18 patients (36\%), but significant abnormalities were only found in 1 subject (2\%). Lumbar puncture was conducted in 3 patients (6\%); all of them had normal cerebrospinal fluid findings. Electroencephalography was done for 30 patients $(60.0 \%)$, and 17 of them (34\%) had abnormal findings such as high-amplitude sharp waves or spike complexes found at the frontal, temporal and/or parietal leads. Head CT scan was done for 34 patients $(68 \%)$, brain MRI for $6(12 \%)$ and 10 patients (20\%) had both of these neuroimaging studies. The CT and MRI studies did not reveal any abnormalities in 34 of the patients (68\%). Sixteen (32\%) were found to have abnormal neuroimaging scan results; however, only 1 of these was con- 
Table 3. Findings of the investigations

\begin{tabular}{lc}
\hline Investigations & $\mathrm{n}(\%)$ \\
\hline CBC abnormalities & \\
Yes & $4(8.0)$ \\
No & $46(92.0)$ \\
Electrolyte abnormalities & \\
Calcium & $5(10.0)$ \\
Magnesium & 0 \\
Sodium & 0 \\
Glucose & 0 \\
None & $45(90.0)$ \\
Lactate and amino acid abnormalities & \\
Yes & $1(2.0)$ \\
No & $17(34.0)$ \\
Not done & $32(64.0)$ \\
Lumbar puncture & \\
Normal & $3(6.0)$ \\
Abnormal & 0 \\
Not done & $47(94.0)$ \\
Electroencephalography & \\
Normal & $13(26.0)$ \\
Abnormal & $17(34.0)$ \\
Not done & $20(40.0)$ \\
Neuroimaging modality & \\
CT head & $34(68.0)$ \\
MRI brain & $6(12.0)$ \\
Both & $10(20.0)$ \\
Neuroimaging findings & \\
Normal & $34(68.0)$ \\
Abnormal & \\
\hline & $16(32.0)$ \\
\hline &
\end{tabular}

$\mathrm{CBC}=$ Complete blood count.

${ }^{1}$ Only 1 subject out of 16 was considered to have an abnormal CT/MRI scan that was clinically significant.

sidered to have clinically significant abnormal CT scan/ MRI findings. The CT scan of the head of this patient showed hypodensity in the right temporal and frontal lobes, and the brain MRI revealed a high signal intensity in the right frontal and occipital lobes with attenuation of the postcerebral artery (more in the right and middle cerebral artery) along with abnormal vascular anastomosis around the circle of Willis; these were consistent with Moyamoya disease. The clinically insignificant findings (fig. 2) observed in the other 15 patients were: mild brain atrophic changes in $2(4 \%)$, small foci of hypo-/hyper-density or intensity in $6(12 \%)$, mild ventricular dilatation in $(5(10 \%)$, pineal cyst in $1(2 \%)$ and arachnoid cyst in 1(2\%). All of these findings were considered insignificant by the pediatric neurologist or the neurosurgeon.

Neuroimaging for First-Attack Nonfebrile Seizure



Fig. 2. Insignificant findings in neuroimaging studies.

\section{Discussion}

In this study, $32.0 \%$ of the patients had abnormal neuroimaging findings, and only $1(6.2 \%)$ had clinically significant abnormal CT scan and MRI findings. This 1-yearold child was diagnosed with Moyamoya disease after presenting with disorientation and focal attack of seizure, and was moved to the Pediatric Neurosurgery Care Unit where she received antiplatelet therapy and was followed up for possible surgical intervention in the future.

This low finding of $6.2 \%$ of the subjects having significant neuroimaging findings requiring medical or surgical management confirmed the finding of $4 \%$ in the study of Aprahamian et al. [16] that involved 319 patients. Equally important, Maytal et al. [3] reported that $<10 \%$ of children with new-onset, nonfebrile seizures had abnormal emergency neuroimaging findings, but that they did not require any medical or surgical intervention. Taken together, all of these findings seem to indicate that the routine use of neuroimaging studies in this group of pediatric patients is not useful.

However, several recommendations have been made regarding the indication of neuroimaging evaluation for first attacks of nonfebrile seizure in pediatric patients [4, 17-19]. Khodapanahandeh and Hadizadeh [17] suggested the use of neuroimaging studies for children who present with focal seizures, abnormal neurological findings or who are younger than 2 years of age. The AAN guidelines [4] recommend the use of emergency neuroimaging for any child of any age who exhibits a postictal focal deficit (i.e. Todd's paresis) that does not resolve quickly or who has not returned to baseline within sev- 
eral hours after the seizure. In other reports $[4,18,19]$ nonurgent MRI should be considered for any child with a significant cognitive or motor impairment of unknown etiology, unexplained abnormalities on neurological examination, a seizure of focal onset with or without secondary generalization, an electroencephalography result that does not represent a benign partial epilepsy of childhood or primary generalized epilepsy, or in children $<1$ year of age. Unfortunately, following the AAN guidelines, neuroimaging studies were ordered for 47 of the patients (94\%) despite the absence of Todd's paresis. This resulted in performing unnecessary, expensive and, in some cases, high-radiation tests on the children based on our findings. It seems that pediatricians in Kuwait do not follow any specific guidelines about when to order a neuroimaging study for this group of patients, but rather that their decision to do so seems to be based on their own experience. These issues should be thoroughly investigated and addressed in future studies in order to improve pediatric care in Kuwait.
A major limitation of this study was the small number of patients. Also, due to its retrospective nature, it could not provide a causal relationship between the findings of neuroimaging studies and the clinical picture of the patients.

\section{Conclusion}

In this study, neuroimaging studies were not useful in evaluating pediatric patients who presented with a first attack of unprovoked, nonfebrile seizures. We therefore recommend that neuroimaging should not be routinely performed in this group of patients unless there is evidence of focal neurological deficits.

\section{Disclosure Statement}

There were no conflicts of interest.

\section{References}

$>1$ Booth TN: Imaging strategies for new onset seizures. Pediatr Radiol 2009;39:S236-S238.

$\checkmark 2$ Long L, Adams C: Evaluation of a single seizure: guidelines for advance practice nurses. J Am Acad Nurse Pract 2000;12:141-145.

$>3$ Maytal J, Krauss JM, Novak G, et al: The role of brain computed tomography in evaluating children with new onset of seizures in the emergency department. Epilepsia 2000;41: 950-954.

$\checkmark 4$ Hirtz D, Ashwal S, Berg A, et al: Practice parameter: evaluating a first nonfebrile seizure in children: report of the quality standards subcommittee of the American Academy of Neurology, the Child Neurology Society, and the American Epilepsy Society. Neurology 2000;55:616-623.

5 Hirtz DG: Generalized tonic-clonic and febrile seizures. Pediatr Clin North Am 1989; 36:365-382.

$\checkmark 6$ Reinus WR, Wippold FJ 2nd, Erickson KK: Seizure patient selection for emergency computed tomography. Ann Emerg Med 1993;22: 1298-1303.

$>7$ Yang PJ, Berger PE, Cohen ME, et al: Computed tomography and childhood seizure disorders. Neurology 1979;29:1084-1088.
-8 Resta M, Palma M, Dicuonzo F, et al: Imaging studies in partial epilepsy in children and adolescents. Epilepsia 1994;35:1187-1193.

-9 Shinnar S, O’Dell C, Mitnick R, et al: Neuroimaging abnormalities in children with an apparent first unprovoked seizure. Epilepsy Res 2001;43:261-269.

10 Vining EP, Freeman JM: Management of nonfebrile seizures. Pediatr Rev 1986;8:185190.

11 Berg AT, Shinnar S, Levy SR, et al: Early development of intractable epilepsy in children: a prospective study. Neurology 2001;56: 1445-1452.

12 Al-Rumayyan AR, Abolfotouh MA: Prevalence and prediction of abnormal CT scan in pediatric patients presenting with a first seizure. Neurosciences (Riyadh) 2012;17:352356.

13 Gaillard WD, Chiron C, Cross JH, et al: Guidelines for imaging infants and children with recent-onset epilepsy. Epilepsia 2009;50: 2147-2153.
-14 Warden CR, Brownstein DR, Del Beccaro MA: Predictors of abnormal findings of computed tomography of the head in pediatric patients presenting with seizures. Ann Emerg Med 1997;29:518-523.

15 Engel J Jr; International League against Epilepsy (ILAE): A proposed diagnostic scheme for people with epileptic seizures and with epilepsy: report of the ILAE Task Force on Classification and Terminology. Epilepsia 2001;42:796-803.

16 Aprahamian N, Harper MB, Prabhu SP, et al: Pediatric first time non-febrile seizure with focal manifestations: is emergent imaging indicated? Seizure 2014;23:740-745.

17 Khodapanahandeh F, Hadizadeh H: Neuroimaging in children with first afebrile seizures: to order or not to order? Arch Iran Med 2006;9:156-158.

-18 Nordli DR Jr, Bazil CW, Scheuer ML, et al: Recognition and classification of seizures in infants. Epilepsia 1997;38:553-560.

19 King MA, Newton MR, Jackson GD, et al: Epileptology of the first-seizure presentation: a clinical, electroencephalographic and magnetic resonance imaging study of 300 consecutive patients. Lancet 1998;352:1007-1011. 